\title{
Cellular Handover Approaches in 2.5G to 5G Technology
}

\author{
Jahangir Khan \\ Department of computer science \& IT, \\ Sarhad university of science and \\ Information Technology Peshawar \\ Ali Abbas \\ Environmental Protection Society- \\ PPAF, Darbar Saidu sharif Swat \\ 19200, Khyber PukhtunKhwa Pakistan \\ Khisro Khan \\ Directorate of Sciences \& Technology, \\ TF-350 Dean Plaza Peshawar 25000, \\ Pakistan
}

25000, Pakistan

\begin{abstract}
The obstruction in cellular network involves the problem when a mobile user travels from one cell to another during a call. As adjacent cell do not use the same radio channels, a call must be transferred from one radio channel to another when a user crosses the line between the adjacent cells. The process of handover takes place that transfer an ongoing call from one cell to another cell as the user moves through the coverage area of a cellular network. There are several different reasons needed to be known to determine whether a handover is required. The signal strength of the base station, along signal strengths of the surrounding stations as in addition the availability of the channels also needed to be known. The mobile monitors the strength of the base stations in cellular network to know for the status of channel availability and the network make decision about the handover. The main goal of this research is to investigate the handover research issues and developing schemes which can handle handovers traffic in order to support on-going calls when mobile users are switching between base stations. The final outcomes of the research will theoretical and mathematical approaches to convince the users as possible to provide effective methods for evaluating handover mechanisms as well as cellular network performance.
\end{abstract}

\section{General Terms}

BSC, MSC, Base stations, LTE, ITU, FDD, TDD

\section{Keywords}

Handover, Hands-off, Next Generation, Signal strength, cell

\section{INTRODUCTION}

Due to rapid change in technology the demand for better and faster cellular communication also increases. This growth in filed of cellular communication has led to increase intensive research toward and development toward cellular system. The main reason of this growth is newly concept of mobile terminal and user mobility. The main characteristics of cellular communication system offer user maximum freedom of moment while using cell phones (mobiles). A cellular network is made up of numbers of cells (or radio cells). Each cell is allocated a band of frequencies and served by base station consisting of transmitter, receiver and control unit. Adjacent cells are assigned different frequencies to avoid interference or cross talk [9]. As more customers use the cellular network with single base station traffic may be build up so there are not enough frequency bands assigned to a cell to handle its calls. An approach can be used to cope with this situation to use the same radio frequency can be reused in different area for a completely different transmission [33]. The degree of reuse determined by how apart cells must be reuse the same frequency is depending upon the actual implementation of the radio link. The reuse of frequencies in different cells is a form of space division multiple access and it requires that location of each mobile agent to be known this is provided through a service known location management or mobility management. In handover process cellular network automatically transfer a call from one radio channel to another radio channel while maintaining quality of services (QoS) of a call [16]. Handover mechanism is extremely important in mobile network because of the cellular architecture employed to maximize spectrum utilization. The number of cell boundaries increases because smaller cells are deployed in order to meet the demand of increased capacity. Each handover require network resources to route the call to next base station. If handover does not occur at right time the QoS may be drop below an adequate level and connection will be dropped [11]. There are several different reasons needed to be known to determine whether a handover is required. The signal strength of the base station, along signal strengths of the surrounding stations. In addition the availability of the channels also needed to be known. The mobile monitors the strength of the base stations, but only the cellular network knows the status of channel availability and the network make the decision about the handover.

For the past five, the wireless infrastructure market has gradually shifted towards 3G technologies (WCDMA/ HSPA, CDMA2000 EVDO Rev. A) globally as consumer demand for more data intensive software applications has increased, driven by a new generation of smart phones like Apple's iPhone. As mobile networks are trying to understand the implications of consumer mobile data usage, networks have buckled under the strains as more and more smart phones continue to access the network. According to the Global mobile Suppliers Association, there are already 64 network operator commitments across 31 countries for LTE technology. We expect that this number will increase dramatically between now and 2013. As for the "other" 4G technology WiMAX, we believe that it will be relegated to a lower level of importance for the ecosystem as global demand, while incrementally increasing, will be overshadowed by the emergence of LTE. LTE actually is considered pre-4G as LTEAdvanced (LTE-A) is the official designated 4G technology by the International Telecommunications Union (ITU) which handles all standards definitions. Current LTE is capable of downlink speeds of $100 \mathrm{Mbps}$ using channel bandwidths of up to $20 \mathrm{MHz}$ while LTE-A technology will support $1 \mathrm{Gbps}$ downlink speeds using channel bandwidths up to $100 \mathrm{MHz}$. The market is not expected to see LTE-A technology emerges until 2014-2015. Since LTE needs a minimum of $10 \mathrm{MHz}$ and optimally $20 \mathrm{MHz}$ of channel bandwidth, currently designated spectrum is unable to 
effectively handle this. There are also two versions of LTE technology: frequency division duplex (FDD) and time division duplex (TDD). The TDD-LTE version is what China Mobile is expected to migrated its current TD-SCDMA technology to. The current users of WiMAX technology such as Clear wire in the US market have also expressed interest in using TDD-LTE to replace WiMAX. As new technologies such as HSPA+, 2C-HSPA+, 4CHSPA+, LTE and LTE-A are introduced, they all have one thing in common, an ever increasing requirement for more channel bandwidth. As such, the regulatory agencies and industry has looked to new spectrum to accommodate this. The $2600 \mathrm{MHz}$ bandwidth is currently un-occupied and can offer $20 \mathrm{MHz}$ channels to mobile operators for LTE operation. This is a new frequency band of operation for the industry and as it is higher in frequency than current $3 \mathrm{G}$ spectrum at $2100 \mathrm{MHz}$, it offers more opportunity for the industry as all deployments in this frequency band are Greenfield. The majority of LTE networks will be deployed in this frequency band. In New BTS Technology the base station has essentially stayed the same without any major changes for the past 20 years. However, the industry has recently begun migration towards a split architecture utilizing a baseband unit (BBU) and remote radio unit (RRU). Essentially, all of the RF functionality of the base station (transceiver, power amplifier, and duplexer filter) has been removed from the cabinet and into a separate unit called the remote radio. The remaining digital, timing and network functionality of the base station is now housed in the BBT. The interface between the RF unit and the digital baseband unit has changed from a coaxial cable to fiber optic cable. The inherent 2-3dB loss of the coaxial feed cable has essentially been eliminated. The RRU is now deployed at the top of the tower mast near the RF antenna while the BBU sits within the BTS shelter or BTS cabinet at the bottom of the tower. The next evolution of this architecture further integrates the RRU with the antenna to create an active smart antenna system called an integrated radio antenna (IRA). There are multiple opportunities near term and within the next decade as the wireless industry transitions to new technologies. We highlight some of the issues that are key enablers and drivers for the market in 2010 and 2011.

New Air Interface LTE (FDD/TDD) and HSPA+

\section{New Spectrum}

2600MHz Auctions and Digital Dividend Spectrum-European and

Asian Analog/ Digital TV Transition 790- 862MHz

900MHz W-CDMA

$700 \mathrm{MHz}$ in US

\section{$2300 \mathrm{MHz}$ in China}

\section{New BTS Technology}

Integrated Radio Antennas (IRA)

Remote Radio Units (RRU)

Multi-Standard Radios (MSR)

\section{New Millimeter wave Radio Technology}

\section{E-Band Gb PTP Radios [12].}

The paper proceed in a pattern that section 2 describes handover initiation, section 3 describes node handover type, section 4 briefs in details with subsections for call handling mechanism, second last section describes the analysis of handover mechanism and last one section concludes the paper.

\section{HANDOVER INITIATION}

Handover initiation is the process of deciding when a request to a handover. Handover is based on received signal strength (RSS) from the current base station and the neighboring base station. The figure 2.6.1 shows a mobile station is moving from one BTS (named BTS1) to another BTS (named BTS2). The RSS of BTS1 decreases as the mobile station moves away and increases as the mobile station get closer to the BTS2 as a result of the signal propagation. Form the below figure 1. we examine various approaches to handover initiation.

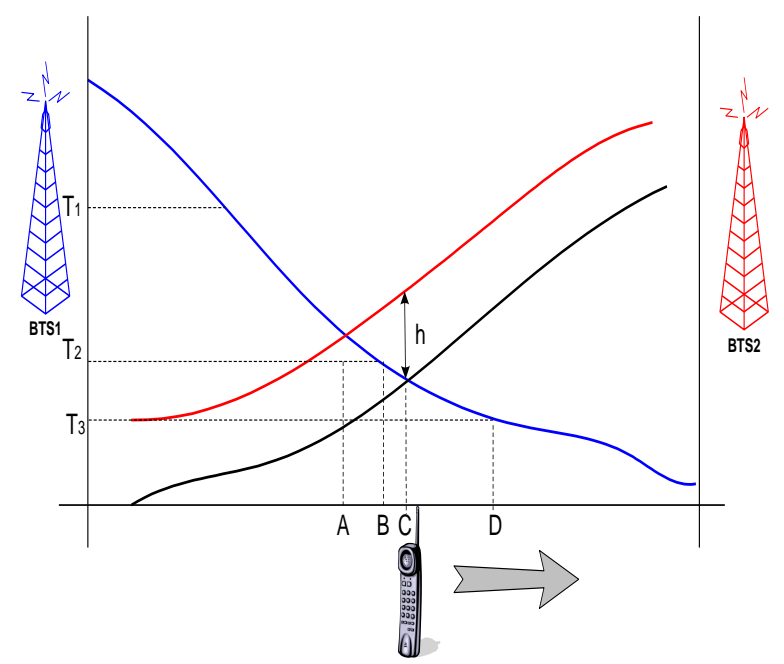

Figure 1. Movement of the MS between two Adjacent BTS for Potential Handover.

Relative Signal Strength: In the relative signal strength the RSS are measured over time and the base station BTS with the strongest signal is chosen to handover. Form the above figure the mobile station is handed of form the BTS1 to BTS2 when the signal strength at BTS2 first exceed that a BTS1. If the signal strength at BTS2 subsequently decreases that of BTS1 the mobile station is handed back toBTS1 and handover occur at point A. At point A signal strength to BTS1 still enough but decreasing. As the signal strength fluctuate due to the multi path effect with averaging power. Due to these signal fluctuation several handover occurs while BTS1 receive signal strength is still sufficient to serve the mobile station. These unnecessary handover can lead to the ping-pong effect in which the mobile station is repeatedly passed between two BTS [44].

Relative Signal Strength with Threshold: This method allows a mobile station to handover if the current BTS is sufficiently weak (less than the predefined threshold) and the other signal is stronger of two. The meaning is as long as the signal at the current BTS is adequate then handover is unnecessary. If the threshold is higher such as T1, in this scheme performs this scheme perform exactly same the relative signal strength scheme, so the handover occur at position A. If the threshold is lower than the value $\mathrm{T} 2$ then the mobile station will delay the handover until the signal level crosses the threshold at position B. If the threshold is set quite low compared to the crossover signal strength such as T3 then the 
mobile station may be drift into the new cell T4. This reduces the quality of the communication link from the BTS1 and may produce in a dropped in call. In addition it produces interference to the co-channel users. A threshold should not be used alone because it $\mathrm{s}$ effectiveness depend upon on prior knowledge of the crossover signal strength between the current and candidate BTS [5].

Relative Signal Strength with Hysteresis: In this scheme handover occurs to new BTS only if the new base station is sufficiently stronger (by a hysteresis $\mathrm{H}$ than the current BTS. In this the handover occurs at point $\mathrm{C}$. This technique prevents the ping pong effect because once handover occurs and the repeatedly handover between BTS is prevented by the hysteresis margin. The only disadvantage of this scheme is that the first handover may still be unnecessary if the BTS1 has sufficient signal strength.

Relative Signal Strength with Hysteresis and Threshold: In this scheme the handover occur by the mobile station to new BTS only if the current signal level drop below the threshold and the targeted base station is stronger than the current one by hysteresis margin. Handover occurs at point $\mathrm{C}$ if the threshold is either $\mathrm{T} 1$ or $\mathrm{T} 2$ and at $\mathrm{D}$ if the threshold is $\mathrm{T} 3$.

Prediction Approaches: There are several prediction techniques developed which base the handover decision on the expected future value of the received signal strength. These techniques are shown through the simulation as they have produced a smaller number of unnecessary handover than both of the relative signal strength and the relative signal strength with hysteresis and the threshold methods [16].

\section{HANDOVER TYPES}

There are different categories of cellular handover which involves different parts of the GSM network. Changing cells within the same BTS is not complicated as the changing of the cell belonging to different MSC. There are mainly two reasons for this kind of handover. The mobile station moves out of the range station or the antenna of BTS respectively. Secondly the wire infrastructure the MSC or the BSC may decide that the traffic in one cell is too high and move some to other cells with lower load. These are the main reasons that initiate different kinds of handover. Following are the different kinds of handover and their details.

Intra-cell BTS Handover: The terms intra-cell and intra BTS handover are used both for frequency change. There is a slight between them but usually they are considered the same. The term intra-cell handover in not real as it deals with the frequency change of a going call. The frequency change occur when the quality of the communication link degrading and the measurements of the neighboring cells better than the current cell. In this situation the BSC which controls the BTS serving the MSC order the MSC and BTS to switch to another frequency which offers better communication link for the call. The communication link degradation is caused by the interference as the neighboring cell using the same frequencies and its better to try another channel. In the intra BTS handover cell involved are synchronized. In the synchronization the MSC sends four HND_ACC messages to the serving BTS. The HND_ACC message is only one byte long and contains the handover reference. The BSC send the HND_CMD to initiate the intra BTS handover. The BSC HND_CMD the time slot and the new channel. The connection is established between the BSC and MSC by exchanging these messages. After these messages exchanged MSC receives HND_PREF message that the handover is performed by the BSC and then BSC requests BTS to release the resources that is no longer used. This synchronized handover saves resources and faster than non synchronized handovers [35].

Intra-BSC Handover: The intra-BSC handover is performed when the MSC changes the BTS but not the BSC. The intra-BSC handover is entirely carried out by the BSC, but the MSC is notified when the handover has taken place. If the targeted cell is in different location area then the MSC needs to perform the location updates procedure after the call. In the intra-BSC handover both synchronized and non synchronized handover are possible. The figure 2 . shows the intra-BSC situation.

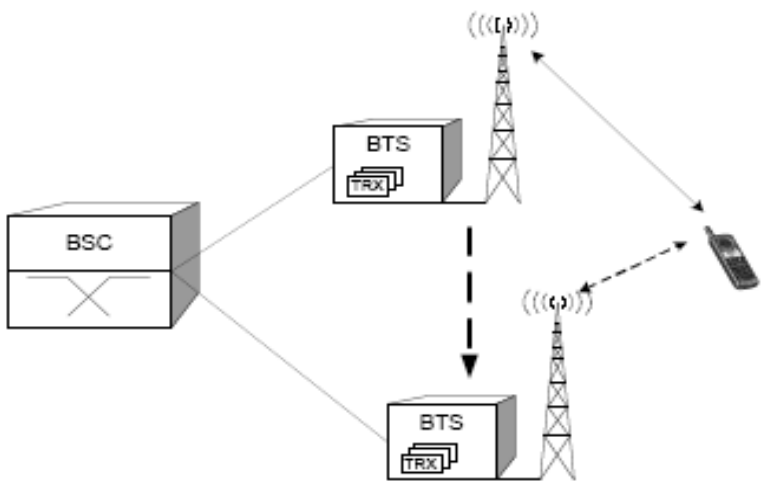

Figure 2. Intra-BSC Handover.

In the intra-BSC handover the BSC send HND_CMD message which contain the time slot, the frequency of the new channel and how MSC shall identify itself on the new channel. And at the same time BTS send PHYS_INFO message and wait for the SABM (Set Asynchronous Balance Mode) from the MSC. When the MSC receives PHYS_INFO message then send SABM in order to establish LAPD for mobile. When the BTS receives the SABM it sends an empty as an acknowledgement to the BSC. Now the MSC only receive the information that the handover was performed by the BSC and BTS releases the used radio resources [35].

Intra-MSC Handover: In the intra-MSC handover when the $\mathrm{BSC}$ decides that handover is required but the targeted cell is controlled by different BSC then it needs assistance from the connected MSC. In comparison to the pervious handover discussed the MSC mandatory for this kind of handover. Responsibilities of the MSC do not include processing the measurements of the BTS or MSC but to conclude the handover. This kind of handover can be other intra-MSC or Inter-MSC. In the intra-MSC handover the targeted cell is allocate in different BSC connected by the same MSC. The MSC contacts the targeted $\mathrm{BSC}$ for allocation of the required resources and inform the BSC when they are ready. After the successful resources allocation the MSC instructed to access the new channel and the call is transferred to the new BSC as shown in the figure 3. 


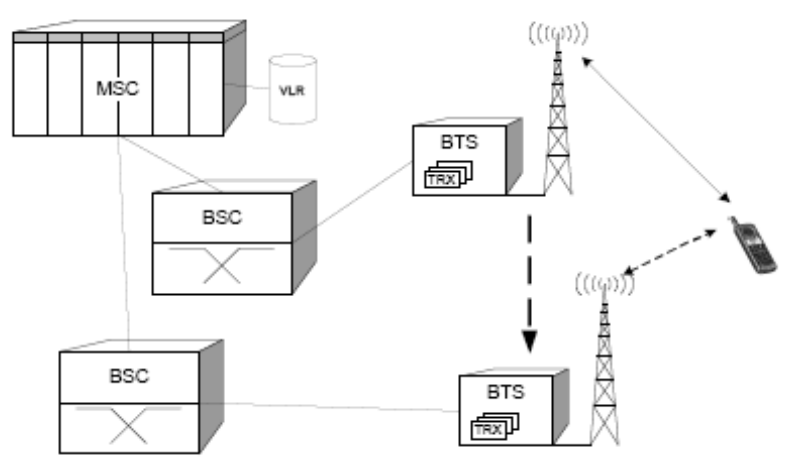

Figure 3. Intra-MSC Handover.

As in the intra-MSC handover the targeted cell may lay outside of the current BSC hence the BSC has to inform the connected MSC. The old BSC sends HND_RQD message to the MSC containing list of all possible target cells for the handover. In this situation the BSC has no knowledge that the BTS belong to the same MSC or different one. It means that the BSC does not know whether an intra-MSC handover or inter-MSC handover. The MSC examines the HND_RQD to the target BSC which assign the channel to be used and identify the BTS. The new BSC then allocate a channel with in the target BTS. If the BSC confirms channel allocation then the targeted BSC writes the HND_CMD message and sends HND_REQ_ACK message back to MSC. The MSC passes the HND_CMD message received from BSC through the old BSC to the MSC containing data identification of the MSC, new BTS target of the handover with the targeted frequency, specified time slot and synchronization. The BTS also sends PHYS_INFO message and waits for SABM from the MSC and does not stop until HND_ACC are received. When the MSC receives PHYS_INFO its stop sending messages and sends SABM to the BTS to establish LAPD for mobile station. When the BTS receives $\mathrm{SABM}$ then the new BSC terminates the handover process. MSC sends CLR_CMD to the old BSC to request to release the radio resources. At the end the SCCP (Signaling Connection Control Part) connection to old BSC terminates and all the resources are released [37].

Inter-MSC Handover: The inter-MSC handover is performed when the two cells belonging to different MSC in the same system. In the inter-MSC handover the targeted cell is connected is connected to different MSC (named as MSC-B) than the one currently serving the call MSC (named as MSC-A). Figure 4. shows inter-MSC handover scenario.

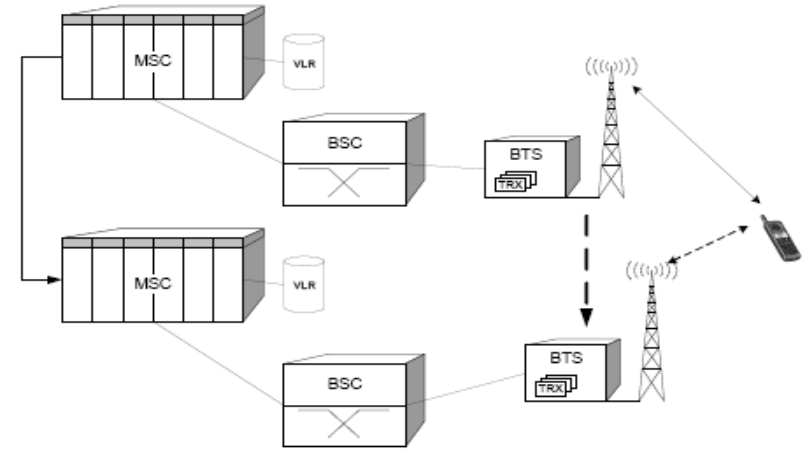

Figure 4. Inter-MSC Handover.
When BSC-A determines that a handover is into another area its sends HND_RQD to its connected MSC-A. HND_RQD contain information the CI (cell identify) and the LAC (location area code) belonging to another MSC area. After identifying the correct neighbors MSC sends prepare handover to MSC-B. The VLR of the MSC-B assign the temporary handover number and passes the HND_REQ to the target BSC-B. In the response of HND_REQ message BSC-B sends HND_REQ_ACK if the resources are available to MSC-B. The MSC-B forward to prepare for handover by sending BSSAP message back to MSCA. In the response MSC-A sends IAM which contains the handover number between MSC-A and MSC-B and after getting the correspondent ACM from MSC-B, MSC-A sends HND_CMD message to MSC-B to perform the handover to the target BTS. The BSC-B sends HND_DET that HND_ACC was received from the MSC. The HND_DET message is forwarded in a MAP to MSC-A. Now the traffic channel is established between MSC-A and MSC-B, and the transport of the payload is carried to MSC-B, and the handover number is released by VLR in MSC-B. By receiving HND_CMP at MSC-B is signaled to MSC-A in a MAP message in SendEndSiganl. This triggers MSC-A to send CLR_CMD message to BSC-A to release the radio resources [37].

\section{CALL HADLING MECANISM}

Handover in cellular network are very important to maintain the quality of a call. In this section a couple of enhancements which can improve the performance of $4 \mathrm{G}$ handover algorithms are presented and studied.

\subsection{Conventional Handover Mechanism}

In cellular network both the mobile station and the BTS regularly measures the radio signal strength. The mobile station transmits its measurements reports continuously to the BTS. If the BTS detects a decrease in radio signal under a minimal level $\mathrm{d}_{\text {urge, }} \mathrm{cf}$ it initiates a handover request as shown in figure 5. The BTS then informs the BSC about the request, which then verifies if it is possible to transfer the call into a new adjacent cell. Actually the BSC checks weather a free channel is available in the new adjacent cell or not. In this situation the BSC does not differentiate between the channel requests either for fresh call or handover. If a free channel is available in the new adjacent cell then handover request can be satisfied, and the mobile station switch to new cell. If there is no free channel in the adjacent cell then it increases the dropping probability of handover call. The drawback of this handover procedure is the fact that the handover request for channel is same as used for fresh calls cf. In conventional handover mechanism is very problematic from the users quality of service perspective, since user can much prefer block a fresh call rather than to be dropped a call in the middle of transmission [26].

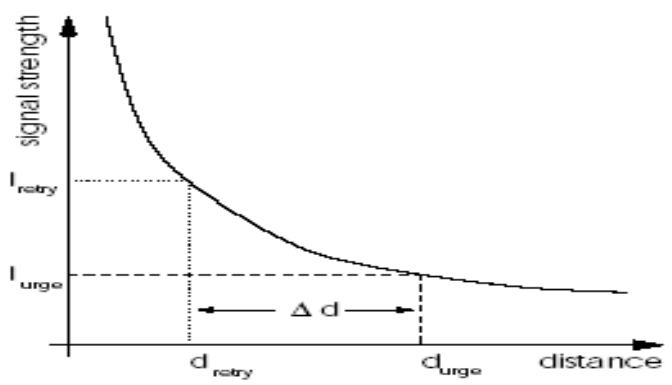

Figure 5. Signal Levels for Handover 


\subsection{Channel Carrying Handover Mechanism}

The channel carrying mechanism allows a mobile station to carry its current channel from one cell to another when it moves across the boundaries under specific conditions. The channel carrying mechanism using a linear cellular system model in which cells or BTS are arranged in linear configuration with minimum reuse distance $r$ as shown in the figure 6 . Suppose $\mathrm{N}$ be the total number of channels available for use in cellular system. Two cells can use the same set of channel as they are apart by distance $r$.

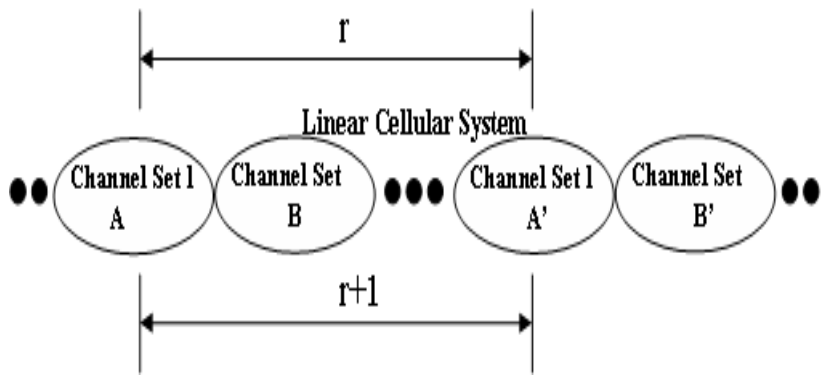

Figure 6. $r$ and $(\mathbf{r}+1)$ Channel Carrying.

To avoid the co-channel interference an advance solution is proposed in which the distance of identical sets of channels is increased to $r+1$ instead of $r$. The distance $r$ is the minimum reuse distance or reuse factor. According to the figure the total number of available channels in each cell is now reduced by amount of $\mathrm{N} / \mathrm{r}+1$ where $\mathrm{N}$ is the total number of available channels. In typical situation where the smaller the reuse distance the more amount of channels is to be lost. The channel carrying mechanism does not require the complex power control or global channel coordination which simplifies its implementation. Handover request are greatly favored over new calls compared to the Conventional handover mechanism. The main drawback of this handover procedure is not suitable for metropolitan environment due to the great amount of channels lost [24].

\subsection{Handover Prioritization Schemes}

Different ideas and approaches are proposed to reduce the handover dropping probability. One approach is to reduce the handover failure rate is to prioritize handover call over new calls. Handover prioritization schemes have a significant impact on the call dropping probability and call blocking probability. Such scheme permits high utilization of bandwidth while guaranteeing the quality of service of handover calls. Basic method of handover prioritization schemes are guard channels (GC), call admission control (CAC) and handover queuing schemes. Some times these schemes are combined together to obtain better results [36].

\subsection{Guard Channel Prioritization Scheme}

The guard channel scheme was introduced in 80s for mobile cellular systems. However the guard channel scheme are still used in telecommunications with the name of Cutoff Priority Schemes. GC scheme improving the probability of successful handover by simply reserving a number of channels exclusively for handover in each cell. The remaining channels can be shared equally between handover and new calls. GC are established only when the number of free channels is equal to or les than the predefined threshold $g$ as shown in figure 7. In this situations fresh calls are bypassed and only handover request are served by the cell until all channels are occupied. The GC scheme is feasible because new calls are less sensitive to delay than the handover calls [19].

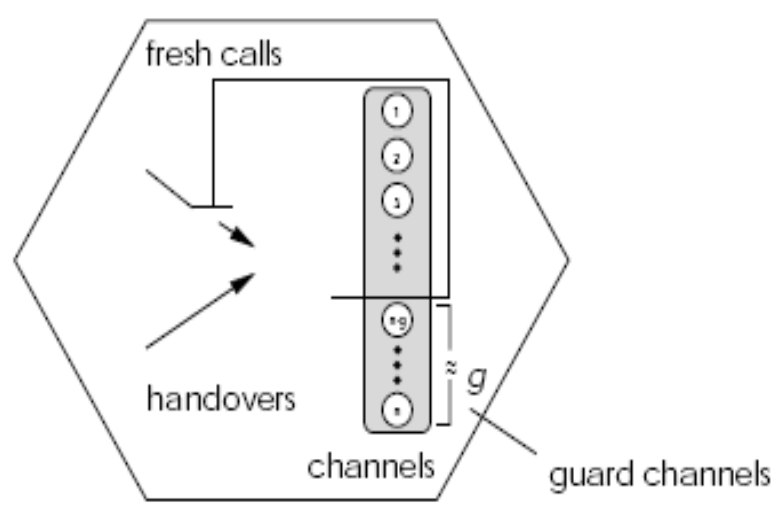

Figure 7. Guard Channels for Handover Request.

If we consider a cellular network with $\mathrm{C}$ the total number of channels in a given cell. According to GC scheme reserve channels for handover are $\mathrm{C}-\mathrm{T}$ where $\mathrm{T}$ is the predefined threshold. The GC will not accept any new call until the channel occupancy goes below the threshold. Suppose the arrival of new and handover call is denoted with $\lambda$ and $v$ respectively. The call holding and call residency for both call is exponentially distributed with $1 / \mu$ and $1 / \eta$ respectively. The total traffic can $b$ e calculated as $\rho=(\lambda+v) /(\mu+\eta)$. Figure 8 . shows the state transition diagram of guard channels.

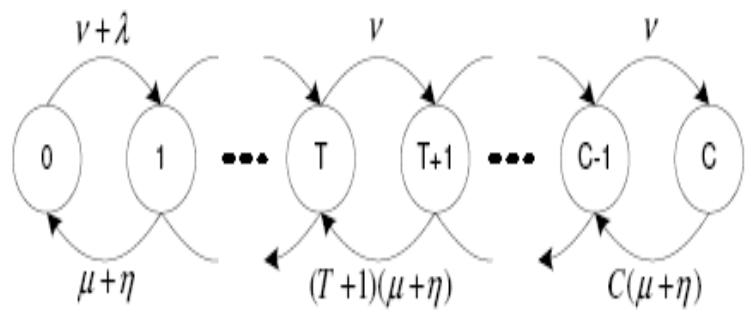

Figure 8. State Transition Diagram of Guard Channels

Therefore according to the cell occupancy by Markov chain it is straight forward to derive the steady state probability $\mathrm{P}_{\mathrm{n}}$ that $\mathrm{n}$ channels are busy [1].

$$
\begin{aligned}
& P_{n}= \begin{cases}\left(\frac{\rho^{n}}{n !}\right) P_{0}, & 0 \leq n \leq T \\
\rho^{T}\left(\frac{\nu^{n-T}}{n !}\right) P_{0}, & T \leq n \leq C\end{cases} \\
& P_{0}=\left[\begin{array}{ll}
\sum_{n=0}^{T} \frac{\rho^{n}}{n !}+\rho^{T} & \sum_{n=T+1}^{C} \frac{\nu^{n-T}}{n !}
\end{array}\right]^{-1}
\end{aligned}
$$

$\mathrm{P}_{\mathrm{b}}=\sum_{\mathrm{n}=\mathrm{T}+1}^{\mathrm{C}} \quad \mathrm{P}_{\mathrm{n}}$ and $\mathrm{pf}=\mathrm{Pc}$. In fact, there is a tradeoff between minimizing $\mathrm{Pd}$ and minimizing $\mathrm{Pb}$. If the number of channels is static chosen then the admission call control fails to satisfy the 
specified Pd. The static channels reservation shows results poor utilization of bandwidth. [13]

To overcome the poor utilization of bandwidth the dynamic guard channel scheme was proposed where the number of guard channels in a radio cell according to the current estimate of handover arrival rate. The handover arrival rate can be derived from the current number of ongoing calls in neighboring cell and the mobility pattern so to keep the handover blocking probabilities close to the targeted objectives while limiting the new call blocking probability to below level. The main characteristic of dynamic GC channel scheme is that it supports any number of classes of traffic each of which can have its own quality of service requirements in the term of channel needed and length of the connection. The GC channel scheme reduce the probability of dropping handover, at the same time the reservation of channel for handover restricts fresh calls from being served and increases their blocking probability to a high level [43].

\subsection{Call Admission Control Prioritization Scheme}

The call admission control scheme refers to the task of deciding whether new call requests are admitted into the network or not. In the CAC the arrival of new call are estimated continuously and if they are higher than the predefined threshold level then some calls are restricted (blocked) irrespective of whether a channel is available or not to decrease the probability of handover calls. In the CAC both the new and handover calls have to access to all channels. If a new call that is generated in cell cannot find an idle channel the call is discarded immediately. There is no queue provided for the new calls to wait [1]

The CAC scheme can be classified into different schemes that consider the local information like (the amount of unused bandwidth in cell where the user currently resides), remote information (the amount of unused information bandwidth in the neighboring cells) or local or remote information to determine weather to accept or reject a call. CAC based on knowledge of both network and user characteristics, keeps the track of available system capacity and accommodates new call request while ensuring quality of service for all existing users. Decisions in $\mathrm{CAC}$ are performed in each BSC in a distributed manner and there is no central coordination. The CAC scheme can be evaluate on the bases of the following properties. [42]

Efficiency: The efficiency of CAC scheme is normally measured in the term of call blocking and call dropping probability. Furthermore the delay of the decision process must be minimized along with the call setup.

Fairness: The fairness of the CAC decision depends upon the individual user arrival, their property for example handover or new call. In the cellular network we can have different subscriber classes each of which different service level agreement can and require different service regulations. It means that the CAC provides differentiating QoS for better network operations.

Stability: The objective of the CAC schemes is to prevent congestion and to ensure the stability for cellular network operations. The CAC reacts too fast to any load change that may lead to unstable control. For example during a call all connection (channel) requests are accepted until congestion occurs and then all the requests are rejected. It is desirable for the cellular network to accommodate as many users into system as possible to maximize the utilization of the radio resources while the QoS for each user must be maintained.

Flexibility: It is important to have a flexible CAC scheme to handle different types of traffic pattern. Traffic patterns in different time for example busy hours with different quality of service requirement. The CAC must be able to reconfigured and extend its support to new traffic regulations.

From the implementation point of view CAC can be divided on the basis of measurements based, centralized, decentralized, locally or globally in the cellular network. This division basically defines different system parameters that are included in the CAC decision for example traffic type, load, cell interference, users requests, and the resources consumptions. Efficient CAC scheme can increase the capacity of the cellular network and the QoS cost-effective. CAC scheme also provides the capability of controlling the load, the group behavior of the traffic classes and user's classes for advanced cellular networks.

\subsection{Handover Queuing Prioritization Schemes}

Queuing handover call prioritization scheme queues the handover calls when all the channels are occupied in the BSC. When a channel is released in the BSC, it is assign to one of the handover call in the queue. The handover queuing scheme reduces the call dropping probability at the expense of the increased call blocking probability and decrease in the ratio of carried to admitted traffic since new call are not assign a channel until all the handover request in the queue are served. [28]

In the handover queuing schemes when the received signal strength of the BSC in the current cell reaches to certain define threshold the call is queued from service a neighboring cell. A new call request is assigned a channel if the queue is empty and if there is at least of free channel in the BSC. The call remains queued until either a channel available in the new cell or the power by the base station in the current cell drops below the receiver threshold. If the call reaches the receiver threshold and no free channel if found then the call is terminated. Queuing handover is possible due to the overlap regions between the adjacent cells in which the mobile station can communicate with more than one base station. This makes provision of the queuing the handover requests for certain time period equal to the time of the mobile host existence in the overlapping area [15].

Queuing is effective only when the handover requests arrive in groups and traffic is low. First in first out (FIFO) scheme is the most common queuing scheme where the handover requests are ordered according to their arrival. To analyze this scheme it is necessary to consider the handover procedure in more detail. By assuming the FIFO queuing strategy and infinite queue size at the base station is assumed as shown in figure 9 .

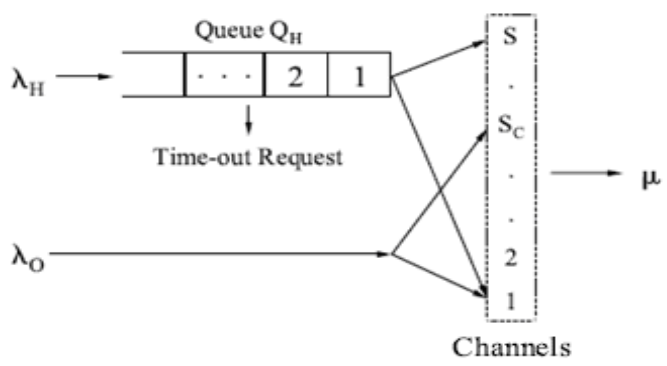

Figure 9. Priority Queue System Model for Handover Call. 
The handover of the mobile station depends on the system parameters such as moving speed, the direction of the mobile station and the cell size. Suppose the state $i(i=0,1,2 \ldots, \infty)$ of a cell as the sum of the channels being used and the number of the handover call request in the queue. Then it is clear form the Markov chain that $i$ one- dimensional. The blow figure shows the sate transition diagram of the cells [44].

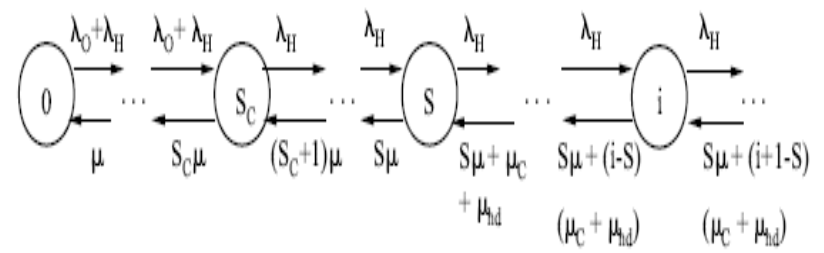

From the above equilibrium probabilities are related to each other through the following equations.

$$
\begin{array}{ll}
i \mu P(i)=\left(\lambda_{O}+\lambda_{H}\right) P(i-1) & 0 \leq i \leq S_{C} \\
i \mu P(i)=\lambda_{H} P(i-1) & S_{C}<i \leq S \\
{\left[S \mu+(i-S)\left(\mu_{C}+\mu_{h \text {-dwell })}\right] P(i)=\lambda_{H} P(i-1)\right.} & S<i \leq \infty
\end{array}
$$

Then the steady state probability is found as follow according to the Markov chain

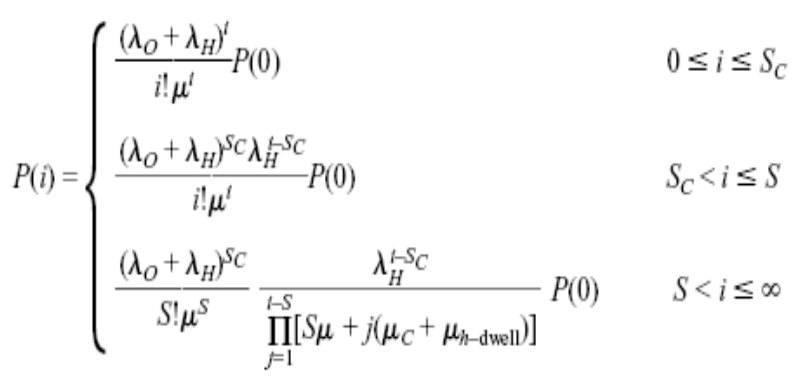

Where

$$
\begin{aligned}
P(0)= & \left\{\sum_{i=0}^{s_{C}} \frac{\left(\lambda_{O}+\lambda_{H}\right)^{t}}{i ! \mu^{t}}+\sum_{i=S_{C}+1}^{S} \frac{\left(\lambda_{O}+\lambda_{H}\right)^{S_{C}} \lambda_{H}^{t-S_{C}}}{i ! \mu^{t}}\right. \\
& \left.+\sum_{i=S+1}^{\infty} \frac{\left(\lambda_{O}+\lambda_{H}\right)^{S_{C}}}{S ! \mu^{S}} \frac{\lambda_{H}^{i-S_{C}}}{\prod_{j=1}^{i-S}\left[S \mu+j\left(\mu_{C}+\mu_{n-\text { dwell }}\right)\right]}\right\}^{-1}
\end{aligned}
$$

Therefore the blocking probability Bo for an originating call is

$$
B o=\sum_{n=s c}^{s} \mathrm{P}(\mathrm{i})
$$

and termination probability of the call is

$$
P_{f}^{\prime}=\sum_{k=0}^{\infty} P(S+k) P_{f h \mid k}
$$

In the FIFO handover Prioritization scheme the probability of the forced termination is decreased however the handover call may be dropped because the handover request can only wait until the receiver threshold is reached. [44]

Some new queuing schemes like Measurement based prioritization scheme (MBSP), very early assignment (VEA), early assignment (EA) and most critical first (MCF) are proposed to improve the performance to improve of the handover queuing scheme by modifying the queuing discipline. In the MBSP the handover calls are added to the queue and the priorities of the calls changes dynamically based on the power level they have. The call with the power level close to the receiver threshold has the highest priority this scheme produce better results than the FIFO queuing schemes. Each of these schemes has its advantages and disadvantages in the term of capacity and services. Like the VEA gives the shortest call setup but is most capacity inefficient. [27]

\subsection{Cell Overlapping and Load Balancing Scheme}

In order to improve the handover call prioritization scheme it is advisable to equalize the traffic load over the cells. Traffic reason and directed retry handover make use of this principal. First the new call to be served and if the receiver is able hear a neighboring cell and are not considered in this situation. Traffic reason handover can be used to transfer traffic from one cell to another neighboring when they are closed to the congestion. The traffic reason handover idea is based on the neighboring cell having an overlapping service area. The overlapping service area arises naturally in GSM cellular system especially in small-cell high capacity micro cellular configurations. The small-cells are capture by subdividing a congested cell each with his own base station. The call arising in the common area (overlapping) of cells have access to channels more than one base station. By appropriate control strategy a cell may select the base station to establish a connation and contribute to efficient spectrum management. By subdividing a congested cell into small- cell the frequency reuse distance is effectively increased which reduce the level of interference and increase the carrier the carrier to interference ratio at both side the mobile station and base station.

Form the previous work it has been proved the directed retry an increase in the overlapping between cells leads to increases the quality of service of the cellular system. A large overlapping area gives more capacity than a smaller overlap, but even by just having a small overlap a significant gain is achieved. The overlap of $0.1 \mathrm{R}$ (where $\mathrm{R}$ is the radius of cell) results an overlapping area equal to $9 \%$ of the cell area gives a gain of at least $6 \%$ whereas if the overlap is equal to $0.5 \mathrm{R}$ means overlapping area is $75 \%$ of the cell area then the capacity gain is boost to $27 \%$. The performance of this functionality is very dependent on the existing overlapping between cells since it is required that at least one neighboring cell has sufficient signal level for the mobile station to be redirected [27].

The higher the overlapping area, the higher the trunking efficiency gain. The overlapping area can be used to reduce the call blocking and dropping probabilities. Mainly two approaches are used to take the advantage of overlapping area, the first one is directed retry where mobile station access the best serving baste station if all the channels are busy in base station then the call is blocked. The second technique which try to balance the load in all the cells. The mobile station accesses the base station as long as 
the load does not exceed a certain predefined threshold. If the load is higher than the threshold and the mobile station is an overlapping area, the mobile station to switch to access the other base station having a low load [2].

By taking one of the simple example where the user positioned is one of the regions formed by the cells as shown in the figure XX. The sizes of the cell me be large enough that not to leave any gap of the coverage area unattended. The mobile station in the overlapping coverage area can be allocated a channel form any of the cell. The size of the overlapping area can be determined by the setting the minimum received power limit and channel to interference ratio. This can be calculated from the link budget and channel o interference ratio values. To take the advantage of the cell overlap user has the choice to connect to any of the base station in the highlighted area. In fact two ways that user can select which base station to connect. The first one is the distance from the base station and the second one is based on the minimum received power threshold [20].

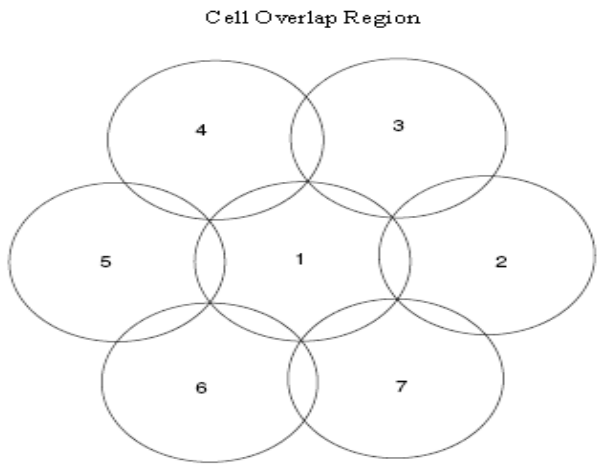

Figure 10. Overlap Areas by Cells.

According to the concept of cell radius when two or more adjacent cells overlap they form a set of individual regions which can be categorized into three types $\mathrm{A}, \mathrm{B}$, and $\mathrm{C}$ according to the number of cell they overlap as shown in figure 10 . These regions can be assigned a channel from one of three cells. The importance of the regions and areas is to perform the channel allocation scheme based on either through the region or area. The number of channels for specific region depends on the size of the regions and specified channel can be used in that area. If we sum of the regions in one cell according to their overlap then they formed a cell area. The blocking probability of the cell can be calculated from those users who are able of choosing a channel from cells A, $\mathrm{B}$, and $\mathrm{C}$ as in figure.11. This maintains the same lowest blocking probability and load balancing in every area [20].

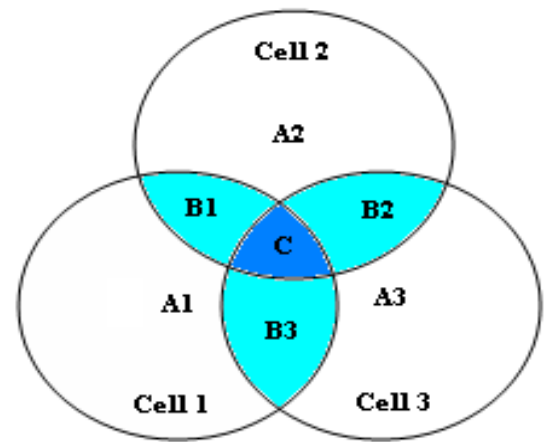

Figure 11. Areas A, B and C of three Cells.

\subsection{Proposed Analysis}

Extensive survey and analysis of the handover prioritization schemes that is guard channels, call admission control and handover queuing has been provided. Furthermore our research indicates that different system uses different schemes to execute the handover mechanism. In this section a couple of enhancements to the handover mechanism are introduced and discussed. The idea of the cells overlap and load balancing scheme which tries to equalize the traffic over cells bas been introduced. It has been analyzed theoretically that capacity depends on the size of the overlapping area between adjacent cells, the numbers of channels per cells and distribution of traffic. The higher the overlapping area, the higher the trucking efficiency gain. The overlapping area can be used to reduce the call blocking and dropping probabilities. The attractive feature of this scheme is that it organizes traffic in distributed manner and doesn't increase the system complexity.

\section{ANALYSIS}

The handover prioritization and hands-off schemes in cellular wireless network provide improved performance at the expense of the increased call blocking probability (CBP). The call blocking probability denotes the possibility of the new channel request being denied into the network from the resources. In handover prioritization schemes an important issue is to limit the call dropping probability (CDP) due to the lack of the resources in the targeted neighbor cell. Both the call blocking and call dropping is the fundamental QoS parameters in cellular wireless network. Other important QoS parameter is the effective channel utilization method which makes effective use of cellular network resources. In this tutorial both the prioritized and the non-prioritized handover scheme are presented. Moreover different prioritization schemes and there extensive classification are presented as well. Non-prioritized scheme handle the both new call and the handover call equally and does not differentiate between them. In this situation the BSC does not differentiate between the channel requests either for fresh call or handover call. The drawback of this handover procedure is the fact that the handover request for channel is same as used for fresh calls. In the non-prioritized scheme the call dropping probability and the call blocking probability are same and are very problematic as the user QoS perspective. Therefore the system must reduce the chances of unsuccessful handovers call by reserving some free channel for handover calls.

All the handover prioritization schemes allocate channels to handovers more frequently than the new call to guarantee the users QoS perspective because new calls are less sensitive to delay than the handover calls. One of the simplest way introduced in the above literature of giving priority to the handover calls is to reserve a number of channels exclusively for the handover in each cell to improve the performance of the cellular system. The guard channel prioritization schemes are established only when the number of free channels is less or equal to predefined threshold. The value of the threshold directly affects the probability of the call blocking and call dropping. According to the cell channel occupancy by Markov chain it is straight forward to derive the steady state probability $\mathrm{P}_{\mathrm{n}}$ that $\mathrm{n}$ channels are busy and then $\mathrm{P}_{\mathrm{b}}=\sum_{\mathrm{n}=\mathrm{T}+1}^{\mathrm{C}} \quad \mathrm{P}_{\mathrm{n}}$ and $\mathrm{pf}=\mathrm{Pc}$. The equation $\mathrm{pf}=\mathrm{Pc}$ shows that the handover failure probability is equal to the call completion probability. 
Several other strategies to allocate channel for the handover request in the queue discipline have been proposed. For example queuing of new call arrivals is possible and is less sensitive regarding the queuing time than the case of handover. Queuing of the new call request shows more improvement than queuing of handover calls. In this scheme new call will be accepted if the number of free channels apart of those reserved for handover is enough for the new request otherwise the call be placed in the queue. As soon as the channel is released by the completing a call or outgoing of the handover request then the new call is served immediately from the FIFO queue. The queuing of the new calls involves the concept of the guard channels and queuing schemes. The performance analysis of queuing new call shows; that the blocking of the handover call decreases with the queuing probability of the new calls and increased in the total carried traffic because new calls will be ultimately served. This scheme also achieves less force termination probability compared to other schemes [19].

\section{Target research approaches}

- Cellular technology

- Optimization techniques

- Mathematical approach for 2.5G-5G

- Handover management

- Market values of new generation technology

- Base stations

- cells

\section{CONCLUSION}

Handover is the procedure that transfers an ongoing call from one cell to another as the user's moves through the coverage area of cellular system. The purpose of the handover procedure it to preserve ongoing calls when the mobile station moving from one cell to another. These measurements reports contain the radio signal quality of the downlink from the BTS to MSC of the call and up to five neighboring cells. The serving BTS measures the uplink from the MSC to BTS radio signal quality of the call and forward in the measurements reports. The information in the measurements reports the BSC is able to decide whether a handover to another cell is needed. These measurements reports are periodically transmitted from the MSC to BSC on the $\mathrm{SACCH}$ channel assigned to each communication for every connection. The repetition duration of the SACCH produces a fixed time grid of $480 \mathrm{~ms}$ in the measurements reports. Handover is based upon that evaluation as the mobile users' leaves the coverage area of the one base station must obtain coverage from the neighboring station in order to keep the connection keep on. Cut off connection or drop call are not acceptable at any level during the call. Handover take places when the traffic level of cell reaches to high level or when neighboring are being underutilized. These performance formulas give foundation after much research into the microcellular handover and overlaid system handover. The sub goal of the research is to get different handover mechanisms that reduce the resources utilizations of the network while reducing the dropping possibilities of the user calls. By proposing new concepts in handover schemes for enhancing and optimizing wireless network performance. Architectural and mathematical approaches proposed here not only for existing cellular technology but also an attempt to $5 \mathrm{G}$ in which we can achieve best QOS along with full and dynamic access to wireless world wide web (wwww) in a very fast and efficient way and also obeys ad hoc mobile networks technology. This paper can make an extraordinary contribution from $2.5 \mathrm{G}$ to $5 \mathrm{G}$ in order to implement the mathematical formulas in a practical world.

\section{ACKNOWLGDEMENTS}

We are very pleased to IJCA and our family support and we also want to thank SARHAD University for their support in our research.

\section{REFERENCES}

[1] Abdulova, V., and Aybay, I. (2006) Handoff Prioritizing Schemes in Cellular Wireless Networks. IEEE Computer Networks Symposiums.16 (18), pp 1-6.

[2] Ahmed, M. (2001) Capacity Enhancement and Performance Analysis of Call Admission and Load Control in Wireless Communication Networks. PhD Dissertation, Carleton University, Canada.

[3] Ariton, E \& Xhafa. (2005) Dynamic Priority Queuing of Handover Calls in Wireless Network: An Analytical Framework. 22(5), June 2004, pp. 904- 916.

[4] Baramatiwala, S. (2004) Global System for Mobile Communications.http://www.cs.wright.edu/ jstephen/ee737/ ResearchPapers/Baramatiwala.doc

[5] Cello. (2001)Cellular Network Optimization Based on Mobile Location.. Finland. http://www.telecom.ntua.gr/cello/

[6] Chaiko, Y. (2004) Simulation of Radio wave Propagation using Propagation Models. http://www.tmf.rtu.lv/DATA_FILES/blank_sc_publication/6 /1001/34/2005_03_02_10_34_11/paper_formatting_Caiko.d oc

[7] Chen, Y. (2003) Soft Handover Issues in Radio Resource. $\mathrm{PhD}$ Dissertation, Queen Mary University, London.

[8] Dirk, H. (2002) The GSM Radio Interface. http://www.aws.cit.ie/personnel/dpesch/notes/msc_sw/GSM_ radio_interface.pdf >

[9] Ekici, E. (1997) Optimal Two-Tier Cellular Network Design. BS Dissertation, Bogazici University, Turkey.

[10] Farely, T. (2006) Early Bell System Overview. http://www.privateline.com/mt_cellbasics/x_appendix/c_earl y_bell_system_overview_of_amps/

[11] Fournogerakis, P., Kyriazakos, S., \& Karetsos, G. (2001) Enhanced Handover Performance in Cellular Systems based on Position Location of Mobile Terminals. Athens, Greece. http://www.telecom.ece.ntua.gr/cello/documents/CELLOWP5-ICCS-PUB02-013-Int.pdf

[12] Earl J. Lum "The Evolution of the BTS Market: Towards 4G Technology" CS MANTECH Conference, May 17th-20th, 2010, Portland, Oregon, USA .

[13] Ghaderi, M and Boutaba. (2006) Call Admission Control in Mobile Cellular Networks: A Comprehensive Survey. Wireless Communications and Mobile Computing, 6(1), pp 69-93. 
http://bcr2.uwaterloo.ca/ rboutaba/Papers/Journals/WCMC04.pdf

[14] Harmantzis, C \&Yaipairoj, S. (2005) Dynamic Pricing with Alternatives for Mobile Network. IEEE. Personal Communications.

http://personal.stevens.edu/ syaipair/A28_1.pdf

[15] IEEE, Handover Schemes in Satellite Networks: State-OfThe-Art and Future Research Directions, NASA, 2006, Volume 8(4).

[16] International Engineering Construction. (2005) Cellular Communications. http://www.iec.org/online/tutorials/cell_comm/topic03.html

[17] J. Scourias. (1997) Overview of the Global System for MobileCommunications.http://ccnga.uwaterloo.ca/ jscouria/ GSM/gsmreport.html

[18] Kapoor, V., Edwards, G., \& Sankar, R. (1994) Handoff Criteria for Personal Communication Networks. Proc. IEEE International Conf on Communication (ICC), New Orleans, PP. 1297-1301.

[19] Katzela, I and Naghshineh. (1996) Channel Assignment Scheme for Cellular Mobile Telecommunication Systems- A Comprehensive Survey, IEEE Personal Communications, 3 (3): pp.10-31.

[20] Katzis, K. (2005) Resource Allocation Techniques for High Altitude Platforms. PhD Dissertation, University of York, UK.

[21] Katzis, K., Pearce, J \& Grace, D. (2004) Fixed Channel Allocation Techniques Exploiting Cell Overlap for High. Altitude Platforms. http://www.elec.york.ac.uk/comms/publications/2004.php

[22] Khesal, N. (2005) Differentiation Strategies for Operators Sharing UMTS Network. Master Thesis, University of Stockholm, Sweden.

[23] Kriegl, J. (2000) Location in Cellular Networks. Diploma Thesis, University of Technology Graz, Australia.

[24] Li, J., Shorff, N and Chong, E (1996) Channel Carrying: A Novel Handoff Scheme for Mobile Cellular Networks. [Online]. ECE Technical Reports. ,http://docs.lib.purdue.edu/ecetr/98

[25] Lutteman, B. (2000) Workforce Diversity How to Manage Workforce Diversity. Master Thesis, University of Stockholm, Sweden.

[26] Mandjes, M., and Tutschku, K. (1996) Efficient Call Handling Procedures in Cellular Mobile Networks, [Online].Report No.144, http://www3.informatik.uniwuerzburg.de/publications/reports.shtml

[27] Nielsen, T., and Wigard, J. (2000) Performance Enhancements in a Frequency Hopping GSM Network. Kluwer Academic Publisher, Netherlands.

[28] Nishitd, D., Jeffrey, N and Vanlandingham, F. (1998) Handoff in Cellular Systems. IEEE Personal Communications. http://www.comsoc.org/pci/private/1998/dec/Tripathi.html>
[29] Oates, B. (2006) Research Information Systems and Computing. SAGE Publisher, UK.

[30] Reyaz, W. (2003) GSM-GPRS-EDGE Technologies. Master Thesis, Department of Computer Engineering Indian Institute of Technology, New Delhi. India.

[31] Scourias, J. (1997) Overview of Global System for Mobile Communications.http://www.shoshin.uwaterloo.ca/ jscouria/ GSM/gsmreport.html

[32] Soy, S. (1997) The Case Study as a Research Method. http://www.gslis.utexas.edu/ ssoy/pubs/index.html>

[33] Stallings, W. (2005) Wireless Communications \& Networks. $2^{\text {nd }}$ Ed. USA, Prentice Hall.

[34] The Mobile Phone Directory. (2006) GSM. http://www.mobile-phonedirectory.org/Glossary/G/GSM.html>

[35] Thomsen, J., and Manggard, R. (2003) Analysis of GSM Handover using Coloured Petri Nets. Master Thesis, University of Aarhus, Denmark.

[36] Tripathi, N., Nortel, Jerry, R and Vanlandingham. (1998) Handoff in Cellular Systems. [Internet] .IEEE Personal Communication.

https://www.cs.tcd.ie/ htewari/papers/tripathi98.pdf

[37] Wei, Y. (2001) Evaluation of Roaming and Download Times in Universal Cellular/Wireless LAN System. Master Thesis Dissertation. Concordia University, Canada.

[38] Wikipedia. (2007) Case Study. [Internet].US http://en.wikipedia.org/wiki/Case_studies >

[39] Wikipedia. (2007) Qualitative Research. http://en.wikipedia.org/wiki/Qualitative_method

[40] Wikipedia. (2007) Time Division Multiple Access.http://en.wikipedia.org/wiki/Time_division_multiple_ access.

[41] Willig, A. (2003) The GSM Air Interface Fundamental and Protocols. $\quad$ http://www.dcl.hpi.unipotsdam.de/teaching/mobilitySem03/slides/hpi_gsm_air_inte rface.pdf

[42] Yliopisto, O. (2005) Smart Packet and Call Admission Control for Efficient Resource Management in Advanced Wireless Networks. PhD Dissertation. University of Oulu, Finland.

[43] Yu, O., and Khanvilkar, S. (2002) Dynamic Adaptive QoS Provisioning over GPRS Wireless Mobile Links. [Internet]. University of Illinois. Chicago. http://mia.ece.uic.edu/ papers/publications/ICC_2002.pdf

[44] Zeng, Q., Agrawal, D. (2001) Handoff in Wireless Mobile Networks..Ohio.USA.http://media.wiley.com/product_data/e xcerpt/28/04714190/ 